\title{
Equilibrium Problems with Applications to Eigenvalue Problems*
}

\author{
Ouayl Chadli ${ }^{\dagger}$ NGai-Ching WOnG $^{\ddagger}$ Jen-ChiH YaO $\S$
}

January 21, 2001

\begin{abstract}
In this paper, we consider equilibrium problems and introduce the concept of $(S)_{+}$ condition for bifunctions. Existence results for equilibrium problems with the $(S)_{+}$condition are derived. As special cases, we obtain several existence results for the generalized nonlinear variational inequality studied by Ding and Tarafdar [12], and the generalized variational inequality studied by Cubiotti and Yao [11], respectively. Finally, applications to a class of eigenvalue problems are given. In particular, we derive an existence result for this class of eigenvalue problems where the parameter does not need to be restricted to bounded intervals and the operator is not needed to be bounded.

Key Words. Equilibrium problems, bifunctions, $(S)_{+}$condition, general nonlinear variational inequalities, generalized variational inequalities, eigenvalue problems.
\end{abstract}

\section{Introduction and Preliminaries.}

Equilibrium problems recently attracts increasing attention and are proven to be significant in the study of optimization, variational inequalities and complementarity problems. The formulation of such problems follows. Given a set $K$ and a bifunction $F: K \times K \rightarrow \mathbb{R}$. We are looking for conditions on $K$ and $F$ to ensure the following is solvable.

$(E P) \quad$ Find $\bar{x} \in K \quad$ such that $\quad F(\bar{x}, y) \geq 0 \quad$ for all $y \in K$.

Many problems of practical interests involve an equilibrium problem formulation in their description, see for example $[5,14,9,1,7]$. Most results on the existence of solutions for equilibrium problems are guaranteed by special algebraic properties on the bifunction $F$, known as generalized monotonicity, see e.g. $[5,4,16,8]$. The current paper is to study equilibrium problems through a topological property that extends a similar one known for variational

${ }^{*}$ This research was supported by a grant from the National Science Council of the Republic of China.

${ }^{\dagger}$ Department of Mathematics, University Cadi Ayyad, Faculty of Sciences Semlalia Marrakesh, Morocco.

${ }^{\ddagger}$ Department of Applied Mathematics, National Sun Yat-Sen University, Kaohsiung 80424, Taiwan, ROC.

${ }^{\S}$ Department of Applied Mathematics, National Sun Yat-Sen University Kaohsiung 80424, Taiwan, ROC. 
inequalities. Some results for mixed equilibrium problems and variational inequalities are established.

As a concrete example of our approach, a class of elliptic variational inequalities involving a $p$-Laplacian type operator is studied. The tools we establish here permits us to improve considerably some recent results in this subject. It seems that our tools is ready to be utilized in other similar problems.

In the following, let $X$ be a Hausdorff topological vector space and $K$ be a nonempty subset of $X$. We shall denote by $X^{*}$ the topological dual of $X$ and by $\sigma\left(X, X^{*}\right)$ (resp. $\sigma\left(X^{*}, X\right)$ ) the weak (resp. weak ${ }^{*}$ ) topology of $X$ (resp. $X^{*}$ ). Let $2^{X}$ be the family of all subsets of $X$. For $A$ in $2^{X}$, let $\operatorname{co}(A)$ be the convex hull and $\operatorname{cl}(A)$ the closure of $A$, respectively.

A bifunction $F: K \times K \rightarrow \mathbb{R}$ is said to be 0-diagonally concave in the second argument [24] if for any finite set $\left\{x_{1}, . ., x_{n}\right\} \subset K$ and any $\lambda_{i} \geq 0, \sum_{i=1}^{n} \lambda_{i}=1$, we have $\sum_{i=1}^{n} \lambda_{i} F\left(y, x_{i}\right) \leq 0$, where $y=\sum_{i=1}^{n} \lambda_{i} x_{i}$. In particular, $F(y, y) \leq 0, \forall y \in K$. Let $T: K \rightarrow 2^{X^{*}} \backslash\{\emptyset\}$, $\eta: K \times K \rightarrow X$ and $g: K \rightarrow X$. The mappings $T$ and $\eta$ are said to have 0-diagonally concave relation [12] if the function $F: K \times K \rightarrow \mathbb{R} \cup\{ \pm \infty\}$ defined by

$$
F(x, y)=\inf _{w \in T x}\langle w, \eta(x, y)\rangle
$$

is 0-diagonally concave in $y$. The mappings $T$ and $g$ are said to have 0-diagonally concave relation [12] if $T$ and $\eta(x, y)=g(x)-g(y)$ have the 0 -diagonally concave relation. The notion of 0-diagonal convexity is defined similarly.

We introduce the following concept of $\left(S_{+}\right)$condition for bifunctions.

Definition 1.1. Let $X$ be a normed space, $K$ a subset of $X$ and $f: K \times K \rightarrow \mathbb{R} \cup\{ \pm \infty\}$ an extended real bifunction. We say that $f$ satisfies the $(S)_{+}$condition if for each sequence $\left\{x_{n}\right\}$ in $K$ such that $x_{n} \rightarrow x \in K$ in $\sigma\left(X, X^{*}\right)$ and $\liminf f\left(x_{n}, x\right) \geq 0$, we have $\left\{x_{n}\right\}$ converges to $x$ in the original topology of $X$.

Remarks. Let $X$ be a Banach space, $K \subset X$ a closed convex subset and $T: K \rightarrow X^{*}$ a nonlinear operator.

1. The notion of operator of class $(S)_{+}$was introduced by Browder [6]. $T$ is said to satisfy condition $(S)_{+}$if for each sequence $\left\{x_{n}\right\}$ in $X$ converging to $x$ in $\sigma\left(X, X^{*}\right)$ and $\lim \sup \left\langle T x_{n}, x_{n}-x\right\rangle \leq 0$, we have the norm convergence of $\left\{x_{n}\right\}$ to $x$. Therefore, if $T$ satisfies condition $(S)_{+}$, then the bifunction $f: K \times K \rightarrow \mathbb{R}$ defined by $f(x, y)=$ $\langle T x, y-x\rangle$ satisfies condition $(S)_{+}$.

2. Let $g: K \rightarrow X$. $T$ is said to satisfy condition $(S)_{+}^{1}$ with respect to $g([10])$ if for each sequence $\left\{x_{n}\right\}$ in $K$ converging to $x$ in $\sigma\left(X, X^{*}\right), T x_{n} \rightarrow x^{*}$ in $\sigma\left(X^{*}, X\right)$ and $\lim \sup \left\langle T x_{n}, g\left(x_{n}\right)\right\rangle \leq\left\langle x^{*}, g(x)\right\rangle$, there is a subsequence of $\left\{x_{n}\right\}$ norm convergent to $x$. We remark that every such sequence $\left\{x_{n}\right\}$ is itself norm convergent to $x$ in this case. 
Indeed, we have the following easy

Proposition 1.2. Let $X$ be a normed space, $K$ a nonempty subset of $X$ and $T: K \rightarrow X^{*}$, $g: K \rightarrow X$ two operators. Suppose that $T(K)$ is bounded and $g$ is weak-norm continuous. Then $T$ satisfies condition $(S)_{+}^{1}$ with respect to $g$ if and only if $f$ defined on $K \times K$ by $f(x, y)=\langle T x, g(y)-g(x)\rangle$ satisfies condition $(S)_{+}$.

\section{Main Results.}

Lemma 2.1. Let $X$ be a Hausdorff topological vector space, $K$ a compact convex subset of $X$ and $G: K \times K \rightarrow \mathbb{R} \cup\{ \pm \infty\}$ such that

(i) for each finite subset $L$ of $K$ and each $y$ in $K$ fixed, the function $x \in K \mapsto G(x, y)$ is upper semicontinuous on $\operatorname{co}(L)$;

(ii) for each finite subset $A$ of $K$, one has $\inf _{y \in \operatorname{co}(A)} \max _{x \in A} G(y, x) \geq 0$.

Then, for each finite subset $Z$ of $K$, there exists $x$ in $\operatorname{co}(Z)$ such that $G(x, z) \geq 0$ for all $z$ in $\operatorname{co}(Z)$.

Proof. Let $A=\left\{y_{1}, \ldots, y_{n}\right\}$ be an arbitrary finite subset of $K$. For each $y$ in $\operatorname{co}(A)$, define

$$
T(y)=\{x \in \operatorname{co}(A): G(x, y) \geq 0\} .
$$

$T(y)$ is non-empty due to (ii). $T(y)$ is closed, since $G(\cdot, y)$ is upper semicontinuous on $\operatorname{co}(A)$. Let us verify that $T: \operatorname{co}(A) \rightarrow 2^{\operatorname{co}(A)}$ is a KKM mapping. Suppose on the contrary that there exists $\left\{z_{1}, \ldots, z_{p}\right\} \subset \operatorname{co}(A)$ and $z \in \operatorname{co}\left(\left\{z_{1}, \ldots, z_{p}\right\}\right)$ such that $G\left(z, z_{j}\right)<0$ for all $i=1, \ldots, p$. It follows that $\max _{i=1, \ldots, p} G\left(z, z_{j}\right)<0$ and therefore

$$
\min _{y \in \operatorname{co}\left(\left\{z_{1}, . ., z_{p}\right\}\right)} \max _{i=1, . ., p} G\left(y, z_{i}\right)<0
$$

which contradicts (ii). Hence by the Fan-KKM Lemma [13], one has $\bigcap_{y \in \operatorname{co}(A)} T(y) \neq \emptyset$. Therefore, for each finite subset $A=\left\{y_{1}, \ldots, y_{n}\right\} \subset K$, there exists $\bar{y}$ in $\operatorname{co}(A)$ such that $G(\bar{y}, y) \geq 0$ for all $y$ in $\operatorname{co}(A)$.

Recall that a strict (LF)-space is the inductive limit of an increasing sequence of Fréchét spaces (See e.g. [17]).

Now we state and prove the main existence result of equilibrium problems in this section.

Theorem 2.2. Let $X$ be a normed space (or more generally, a metrizable locally convex space or a strict (LF)-space). Let $K$ be a convex subset of $X$ and $f, h: K \times K \rightarrow \mathbb{R} \cup\{ \pm \infty\}$ two bifunctions such that $f$ and $h$ do not assume simultaneously $+\infty$ or $-\infty$. Suppose 
(i) for each $y$ in $K, f(\cdot, y)$ is upper semicontinuous and $h(\cdot, y)$ is weakly upper semicontinuous on $K$;

(ii) $f$ and $h$ are 0 -diagonally convex, and $h(x, x)=0, \forall x \in K$;

(iii) $f$ satisfies the $(S)_{+}$condition;

(iv) there exist a nonempty weakly compact subset $W$ and a weakly compact convex subset $B$ of $K$ such that for each $x$ in $K \backslash W$ there exists $y$ in $B$ with

$$
f(x, y)+h(x, y)<0 .
$$

Then, there exists an $\bar{x}$ in $K$ such that $f(\bar{x}, y)+h(\bar{x}, y) \geq 0$ for all $y$ in $K$.

Proof. We first suppose that $K$ is weakly compact. Let us denote by $\mathcal{L}$ the set of all finite subsets of $K$. It follows from Lemma 2.1, (i) and (ii) that for each $Z$ in $\mathcal{L}$, there exists an $x$ in $\operatorname{co}(Z)$ such that

$$
f(x, z)+h(x, z) \geq 0, \quad \forall z \in \operatorname{co}(Z)
$$

Set

$$
M_{Z}=\{x \in K: f(x, z)+h(x, z) \geq 0 \text { for all } z \text { in } \operatorname{co}(Z)\} .
$$

Note that $\operatorname{cl}\left(M_{Z}\right)$ is compact, where the closure is taken in the weak topology $\sigma\left(X, X^{*}\right)$ of $X$. We are going to show that $\bigcap_{Z \in \mathcal{L}} \operatorname{cl}\left(M_{Z}\right) \neq \emptyset$. Since $K$ is weakly compact, it suffices to verify that the family $\left\{\operatorname{cl}\left(M_{Z}\right): Z \in \mathcal{L}\right\}$ has the finite intersection property. Let $Y_{1}, \ldots, Y_{n}$ be in $\mathcal{L}$ and set $Y=Y_{1} \cup \cdots \cup Y_{n} \in \mathcal{L}$. Then the nonempty set $M_{Y} \subset M_{Y_{1}} \cap \cdots \cap M_{Y_{n}}$. Consequently,

$$
\bigcap_{Z \in \mathcal{L}} \operatorname{cl}\left(M_{Z}\right) \neq \emptyset
$$

Let $\bar{x}$ be a common weak cluster point in all such $M_{Z}$. For each $y$ in $K$, we set $Y=\{y, \bar{x}\} \in \mathcal{L}$. Since $\bar{x} \in \operatorname{cl}\left(M_{Y}\right)$, there exists a sequence $\left\{x_{n}\right\}$ in $M_{Y}$ such that $x_{n} \rightarrow \bar{x}$ in $\sigma\left(X, X^{*}\right)$ topology. By construction, we have

$$
f\left(x_{n}, z\right)+h\left(x_{n}, z\right) \geq 0, \quad \forall z \in \operatorname{co}(\{y, \bar{x}\})
$$

Putting $z=\bar{x}$ into (1), we get

$$
f\left(x_{n}, \bar{x}\right)+h\left(x_{n}, \bar{x}\right) \geq 0 .
$$

Therefore,

$$
0 \leq \liminf \left[f\left(x_{n}, \bar{x}\right)+h\left(x_{n}, \bar{x}\right)\right] \leq \liminf f\left(x_{n}, \bar{x}\right)+\lim \sup h\left(x_{n}, \bar{x}\right) .
$$

Since $h(\cdot, \bar{x})$ is weakly upper semicontinuous and $h(\bar{x}, \bar{x})=0$, it follows from (2) that

$$
\liminf f\left(x_{n}, \bar{x}\right) \geq 0
$$


Hence, by the $(S)_{+}$condition on $f$, we derive that $x_{n} \rightarrow \bar{x}$ in norm. On the other hand, by putting $z=y$ into (1), one has

$$
f\left(x_{n}, y\right)+h\left(x_{n}, y\right) \geq 0 .
$$

Taking the limit sup in (3), one deduces

$$
f(\bar{x}, y)+h(\bar{x}, y) \geq \limsup f\left(x_{n}, y\right)+\lim \sup h\left(x_{n}, y\right) \geq \lim \sup \left[f\left(x_{n}, y\right)+h\left(x_{n}, y\right)\right] \geq 0 .
$$

Now, we consider the general case where $K$ is not necessarily weakly compact. For each $L$ in $\mathcal{L}$, we set $C=\operatorname{co}(B \cup L) . C$ is compact since $B$ is compact and convex. By what has already been proved, there exists an $\tilde{x}$ in $C$ such that $f(\tilde{x}, y)+h(\tilde{x}, y) \geq 0$ for all $y$ in $C$. By the coercivity assumption (iv), one has $\tilde{x} \in W$.

Let $\tilde{M}_{L}=\{x \in W: f(x, z)+h(x, z) \geq 0$ for all $z$ in $\operatorname{co}(B \cup L)\}$. It is easy to see that the family $\left\{\tilde{M}_{Z}: Z \in \mathcal{L}\right\}$ has the finite intersection property. Therefore, since $W$ is compact, one has $\bigcap_{Z \in \mathcal{L}} \operatorname{cl}\left(\tilde{M}_{Z}\right) \neq \emptyset$. Let $\bar{x}$ be a common point in all such $\operatorname{cl}\left(\tilde{M}_{Z}\right)$. We claim that $\bar{x}$ is a solution. For this, let $y \in K$ and consider $Z=\{\bar{x}, y\}$. Since $\bar{x} \in \operatorname{cl}\left(\tilde{M}_{Z}\right)$, there exists a sequence $\left\{x_{n}\right\}$ in $\operatorname{cl}\left(\tilde{M}_{Z}\right)$ such that $x_{n} \rightarrow \bar{x}$ in the $\sigma\left(X, X^{*}\right)$ topology. Note that $f\left(x_{n}, z\right)+h\left(x_{n}, z\right) \geq 0$ for each $z$ in $\operatorname{co}(B \cup Z)$. By the same argument above, we have

$$
f(\bar{x}, y)+h(\bar{x}, y) \geq 0,
$$

which completes the proof.

Remarks. In the previous theorem, we would like to make the following observations.

1. The same conclusion and proof remain valid if the underlying space $X$ is just a metrizable locally convex space or a strict (LF)-space. To utilize the $(S)_{+}$condition on $f$ in this case, we shall make use of the following result which can be found in, for example, $[17$, p. 313]: Suppose that $X$ is a metrizable locally convex space or a strict (LF)-space. If $x_{0}$ is a weak closure point of a weakly relatively compact subset $K$ of $X$, then $x_{0}$ is the weak limit of a sequence in $K$.

2. The convex subset $K$ of $X$ is not needed to be closed or bounded. But if $K$ is assumed to be weakly compact then the coercivity condition (iv) is not needed; or else, one can simply make $W=K$.

We are going to improve some recent results in the literature as application of Theorem 2.2. Consider the following general nonlinear variational inequality problem studied by DingTarafdar [12]. Let $K$ be a nonempty closed convex subset of $X$, a real normed space (or more generally a metrizable locally convex space or a strict (LF)-space) with topological dual $X^{*}$. Let $A: K \rightarrow X^{*}, g: K \rightarrow X$ and $a, b: K \times K \rightarrow \mathbb{R}$. Find $\bar{x} \in K$ such that

$$
(G N V I) \quad\langle A \bar{x}, g(\bar{x})-g(y)\rangle \leq a(\bar{x}, y-\bar{x})+b(\bar{x}, y)-b(\bar{x}, \bar{x}) \quad \text { for all } y \in K .
$$

We make the following assumptions: 
$\left[\mathbf{H}_{1}\right] a, b$ are 0 diagonally convex, and $a(x, 0)=0, \forall x \in K$;

$\left[\mathbf{H}_{2}\right] a$ is jointly weakly upper semicontinuous on $K \times K$, and for each $y$ in $K, b(\cdot, y)$ is weakly upper semicontinuous on $K$;

$\left[\mathbf{H}_{3}\right]$ the function $x \in K \mapsto b(x, x)$ is weakly lower semicontinuous on $K$.

One has the following existence result for $(G N V I)$.

Theorem 2.3. Suppose $\left[\mathbf{H}_{\mathbf{1}}\right]-\left[\mathbf{H}_{\mathbf{3}}\right]$ in the above (GNVI) and the following conditions are satisfied.

(i) A is norm-weak* continuous in the sense that the function $k \mapsto\langle A k, y\rangle$ is continuous on $K$ for all $y$ in $X$.

(ii) $g$ is continuous from the norm topology of $K$ to the norm topology of $X$.

(iii) $A$ and $g$ have 0-diagonally concave relation on $K$.

(iv) The bifunction $f: K \times K \rightarrow \mathbb{R}$ defined for $x, y$ in $K$ by $f(x, y)=\langle A x, g(y)-g(x)\rangle$ satisfies condition $(S)_{+}$.

(v) There exist a nonempty weakly compact subset $W$ and a weakly compact convex subset $B$ of $K$ such that for each $x$ in $K \backslash W$, there exists a $y$ in $B$ such that

$$
\langle A x, g(y)-g(x)\rangle+a(x, y-x)+b(x, y)-b(x, x)<0 .
$$

Then, problem (GNVI) has at least one solution.

Proof. Let $f, h: K \times K \longrightarrow \mathbb{R}$ be defined respectively by

$$
f(x, y)=\langle A x, g(y)-g(x)\rangle \quad \text { and } \quad h(x, y)=a(x, y-x)+b(x, y)-b(x, x) .
$$

Suppose a sequence $\left\{x_{n}\right\}$ norm converges to $x$ in $K$. By the norm-weak* continuity of $A$, $\left\{\left\langle A x_{n}, y\right\rangle\right\}$ is bounded for each $y$ in $X$. It follows from the Tychonoff Theorem that $\left\{A x_{n}\right\}$ is $\sigma\left(X^{*}, X\right)$ compact. Consequently, $\lim _{n}\left\langle A x_{n}, g(x)-g\left(x_{n}\right)\right\rangle=0$ as $g\left(x_{n}\right)$ converges in $g(x)$ in norm. Then for all $y$ in $K$,

$$
\begin{aligned}
\lim _{n} f\left(x_{n}, y\right) & =\lim _{n}\left\langle A x_{n}, g(y)-g\left(x_{n}\right)\right\rangle \\
& =\lim _{n}\left\langle A x_{n}, g(y)-g(x)\right\rangle+\lim _{n}\left\langle A x_{n}, g(x)-g\left(x_{n}\right)\right\rangle \\
& =\langle A x, g(y)-g(x)\rangle .
\end{aligned}
$$

Thus $f$ is continuous on $K \times K$. Now Theorem 2.2 applies.

\section{Remark.}

Suppose $A(K)$ is bounded and $g$ is weak-norm continuous. By Proposition 1.2, one deduces that condition (iv) is equivalent to that $A$ satisfies condition $(S)_{+}^{1}$ with respect to $g$. 
Therefore Theorem 2.3 improves the results for the problem (GNVI) in [10]. Furthermore, the subset $K$ is not needed to be closed or bounded and the space $X$ is not needed to be a reflexive Banach space. But when $K$ is weakly compact, the coercivity condition (vi) above is not needed.

We consider next the following generalized variational-like inequality problem. Let $X$ be a normed space with topological dual space $X^{*}$ and $K$ be a nonempty closed convex subset of $X$. Let $T: K \rightarrow 2^{X^{*}}, \eta: K \times K \rightarrow X$ and $h: K \times K \rightarrow \mathbb{R}$.

$(G V L I) \quad$ Find $\bar{x} \in K, \bar{\xi} \in T \bar{x} \quad$ such that $\langle\bar{\xi}, \eta(\bar{x}, y)\rangle+h(\bar{x}, y) \geq 0$ for all $v \in K$.

Definition 2.4. Let $T$ be a multi-valued mapping from a nonempty subset $K$ of a Hausdorff topological vector space $X$ into its topological dual $X^{*}$. Let $\eta: K \times K \rightarrow \mathbb{R}$. $T$ is said to satisfy condition $(S)_{+}$with respect to $\eta$ if for any sequence $\left\{x_{n}\right\}$ in $K$ such that $x_{n} \rightarrow x$ in $\sigma\left(X, X^{*}\right)$ and

$$
\liminf _{n} \sup _{\xi \in T x_{n}}\left\langle\xi, \eta\left(x_{n}, x\right)\right\rangle \geq 0
$$

the sequence $\left\{x_{n}\right\}$ converges to $x$ in the original topology of $X$.

\section{Compare this with}

Definition 2.5. [11] Let $T$ be a multi-valued operator from a nonempty closed convex subset $K$ of a real Banach space $X$ into $X^{*}$. The operator $T$ is said to satisfy condition $(S)_{+}^{1}$ if for any sequence $\left\{\left(x_{n}, f_{n}\right)\right\} \subset K \times X^{*}$ with $f_{n}$ in $T x_{n}$ such that $x_{n} \rightarrow x$ in $\sigma\left(X, X^{*}\right), f_{n} \rightarrow f$ in $\sigma\left(X^{*}, X\right)$ and $\lim \sup \left\langle f_{n}, x_{n}\right\rangle \leq\langle f, x\rangle$, we have that $\left\{x_{n}\right\}$ has a subsequence norm convergent. (Indeed, $x_{n}$ itself converges to $x$ in norm.)

We make the following observation.

Proposition 2.6. Assume that $T(K)$ is bounded. Then, $T$ satisfies condition $(S)_{+}^{1}$ if and only if $T$ satisfies condition $(S)_{+}$with respect to $\eta$ defined by $\eta(x, y)=y-x$.

Theorem 2.7. Let $X$ be a normed space (or more generally, a metrizable locally convex space or a strict (LF)-space) and $K$ be a nonempty convex subset of $X$. Let $T: K \rightarrow 2^{X^{*}}$, $\eta: K \times K \rightarrow X$ and $h: K \times K \rightarrow \mathbb{R}$ such that

(i) $T$ is upper semicontinuous from $K$ into $2^{X^{*}}$, and for each $x$ in $K, T x$ is a nonempty compact subset of $X^{*}$;

(ii) $T$ satisfies condition $(S)_{+}$with respect to $\eta$;

(iii) for each $y$ in $K$ fixed, $h(\cdot, y)$ is weakly upper semicontinuous and $\eta(\cdot, y)$ is continuous;

(iv) for each $x$ in $K$ fixed, $h(x,$.$) is convex with h(x, x)=0$ and the function $\eta(x, \cdot)$ is affine; 
(v) there exist a nonempty weakly compact subset $W$ and a weakly compact convex subset $B$ of $K$ such that for each $x$ in $K \backslash W$ there exists a $y$ in $B$ with

$$
\sup _{\xi \in T x}\langle\xi, \eta(x, y)\rangle+h(x, y)<0 .
$$

Then there exists an $\bar{x}$ in $K$ and $a \bar{\xi}$ in $\operatorname{co}(T \bar{x})$ such that

$$
\langle\bar{\xi}, \eta(\bar{x}, y)\rangle+h(\bar{x}, y) \geq 0 \quad \text { for all } y \in K .
$$

In particular, we have

$$
\sup _{\xi \in T \bar{x}}\langle\xi, \eta(\bar{x}, y)\rangle+h(\bar{x}, y) \geq 0 \quad \text { for all } y \in K
$$

Proof. Redefining $T x$ by $\operatorname{co}(T x)$, we can assume $T$ is convex-valued. We shall apply Theorem 2.2, with $f(x, y)=\sup _{\xi \in T x}\langle\xi, \eta(x, y)\rangle$. Since $\eta(\cdot, y)$ is continuous, from [2, Proposition 21, p.119] and condition $(i)$ one deduces that $f(\cdot, y)$ is upper semicontinuous. On the other hand, one can see that all the other conditions of Theorem 2.2 are satisfied. Hence, there exists an $\bar{x}$ in $K$ such that

$$
\sup _{\xi \in T \bar{x}}\langle\xi, \eta(\bar{x}, y)\rangle+h(\bar{x}, y) \geq 0 \quad \text { for all } y \in K \text {. }
$$

Note that $T(\bar{x})$ is now assumed to be convex. Define the function $p: T(\bar{x}) \times K \rightarrow \mathbb{R}$ by

$$
p(\xi, y)=\langle\xi, \eta(\bar{x}, y)\rangle+h(\bar{x}, y) .
$$

Hence by Lemma 1 in [5], one deduces that there exists a $\bar{\xi}$ in $T \bar{x}$ such that $p(\bar{\xi}, y) \geq 0$ for all $y$ in $K$. This completes the proof.

Remark. If $h=0$ and $\eta(x, y)=y-x$, then one can see, taking into account of Proposition 2.6, that Theorem 2.7 improves Theorem 4.1 in [11], since $T(K)$ is not needed to be bounded and $K$ is not assumed to be a closed subset of $X$.

\section{An application.}

We consider the following eigenvalue problem:

$$
(E V P)\left\{\begin{array}{l}
\text { find } u \in K, \lambda \in \mathbb{R} \text { such that } \\
\int_{\Omega}\langle J(\nabla u), \nabla(v-u)\rangle d x+\int_{\Omega} g(x, u, \lambda)(v-u) d x \geq 0 \text { for all } v \in K,
\end{array}\right.
$$

where $\langle.,$.$\rangle is the inner product in \mathbb{R}^{N},\|$.$\| is the norm defined by the inner product, \Omega$ is an open subset of $\mathbb{R}^{N}$ not necessarily bounded, $g$ is a real function defined on $\Omega \times \mathbb{R} \times \mathbb{R}$ and $K=\left\{u \in W^{1, p}(\Omega): u(x) \geq \tau(x)\right.$ a.e. on $\left.\Omega\right\}, 1<p<N, \tau \in W^{1, p}(\Omega)$, where $W^{1, p}(\Omega)$ is the completion of $C_{0}^{\infty}(\Omega)$ with respect to the norm $\left(\int_{\Omega}\|\nabla u\|^{p}\right)^{\frac{1}{p}}$. J is a function defined 
on $\mathbb{R}^{N}$ as follows: $J(0)=0$ and $J(\xi)=[\alpha(\xi)]^{p-1} \nabla \alpha(\xi)$ for $p>1$ and $\xi \in \mathbb{R}^{N} \backslash\{0\}$ with $\alpha: \mathbb{R}^{N} \rightarrow\left[0,+\infty\left[\right.\right.$, a convex function of class $C^{1}\left(\mathbb{R}^{N} \backslash\{0\}\right)$ satisfying $\alpha(t \xi)=t \alpha(\xi)$ for $t>0$ and $\xi \in \mathbb{R}^{N}$.

Problem $(E V P)$ amounts to finding eigenvalues and eigenvectors of a class of variational inequalities involving a $p$-Laplacian type operator $\operatorname{div}(J(\nabla u))$. Observe that $\Omega$ can be unbounded, and in particular, it can be $\mathbb{R}^{N}$. The $p$-Laplacian operator $\operatorname{div}(J(\nabla u))$ was introduced in [3] and problems similar to $(E V P)$, when $\Omega$ is bounded and $\operatorname{div}(J(\nabla u))$ is the Laplacian operator $\triangle u$ (i.e. $p=2$ and $\alpha(\xi)=|\xi|)$ or $\operatorname{div}(J(\nabla u))$ is replaced by a strongly monotone operator were studied by using the topological degree method and the minimax principle, see $[19,20,21]$. In the case when $\operatorname{div}(J(\nabla u))$ is the $p$-Laplacian operator and $\Omega$ is bounded, problems similar to $(E V P)$ were studied by using the mountain pass theorem and the Ekeland variational principle, see [22, 23]. Another approach was proposed in [25] by using arguments from [26].

Let $X=W^{1, p}(\Omega)$ be endowed with its weak topology $\sigma\left(X, X^{*}\right)$ and $(\cdot, \cdot): X^{*} \times X \rightarrow \mathbb{R}$ the duality paring between $X$ and $X^{*}$. We shall denote by $\|\cdot\|_{L^{p}(\Omega)}$ the norm in $L^{p}(\Omega)$ and by $\|\cdot\|_{X}$ the norm in $X$, i.e. $\|u\|_{X}=\left(\int_{\Omega}\|\nabla u\|^{p} d x\right)^{\frac{1}{p}}$. Note that $K=\{u \in X: u(x) \geq \tau(x)$ a.e. on $\Omega\}$ is a closed convex subset of $X$. Let us consider the nonlinear operator $A$ defined on $X$ by

$$
(A(u), v)=\int_{\Omega}\langle J(\nabla u), \nabla v\rangle d x .
$$

We suppose that the function $J$ satisfies the following assumptions :

$\left[\mathbf{A}_{\mathbf{1}}\right] \quad$ for each $\xi, \eta \in \mathbb{R}^{N}$ one has $\langle J(\xi)-J(\eta), \xi-\eta\rangle \geq \mu(\|\xi\|+\|\eta\|)^{p-2}\|\xi-\eta\|^{2}$;

$\left[\mathbf{\mathbf { A } _ { 2 }}\right] \quad$ for each $\xi, \eta \in \mathbb{R}^{N}$ one has $\|J(\xi)-J(\eta)\| \leq \delta(\|\xi\|+\|\eta\|)^{p-2}\|\xi-\eta\|$.

We suppose that $g: \Omega \times \mathbb{R} \times \mathbb{R} \rightarrow \mathbb{R}$ is of the form

$$
g(x, s, \lambda)=\lambda \alpha(x)|s|^{p-2} s+\theta(x, s),
$$

where $\alpha(x) \in L^{\frac{N}{p}}(\Omega)$ and $\theta(x, s)$ satisfies the following growth condition:

$$
\left[\mathbf{A}_{3}\right] \quad \text { for each } x \in \Omega, s \in \mathbb{R} \text {, one has }|\theta(x, s)| \leq \beta(x)+\gamma(x)|s|^{q-1},
$$

with $1<q<p, \beta \in\left(L^{p^{*}}(\Omega)\right)^{\prime}, \gamma \in L^{\frac{p^{*}}{p^{*}-q}}(\Omega)$ where $p^{*}$ is the critical Sobolev value $p^{*}=\frac{N p}{N-p}$. We shall also assume that

$\left[\mathbf{A}_{4}\right] \quad X$ is compactly embedded in $L^{p^{*}}(\Omega)$.

From the expression of $g$, problem $(E V P)$ becomes

$$
(E V P)\left\{\begin{array}{l}
\text { find } u \in K \text { such that for all } v \in K \\
(A(u), v-u)+\lambda \int_{\Omega} \alpha(x)|u|^{p-2} u(v-u) d x+\int_{\Omega} \theta(x, u)(v-u) d x \geq 0 .
\end{array}\right.
$$


Let us set for $u, v$ in $K$,

$$
\begin{aligned}
& f(u, v)=(A(u), v-u)=\int_{\Omega}\langle J(\nabla u), \nabla(v-u)\rangle d x, \\
& \varphi(u, v)=\lambda \int_{\Omega} \alpha(x)|u|^{p-2} u(v-u) d x \\
& \psi(u, v)=\int_{\Omega} \theta(x, u)(v-u) d x
\end{aligned}
$$

and $h(u, v)=\varphi(u, v)+\psi(u, v)$. Then the problem $(E V P)$ is equivalent to the following equilibrium problem

$$
(E P)\left\{\begin{array}{l}
\text { find } u \in K \text { such that } \\
f(u, v)+h(u, v) \geq 0
\end{array} \quad \text { for all } v \in K .\right.
$$

Lemma 3.1. Assume that $\left[\mathbf{A}_{1}\right]$ is satisfied, then the bifunction $f$ satisfies the $(S)_{+}$condition.

Proof. Let $\left\{u_{n}\right\} \subset K$ such that $u_{n} \rightarrow u$ in $\sigma\left(X, X^{*}\right)$ and $\liminf _{n} f\left(u_{n}, u\right) \geq 0$. One has

$$
\begin{aligned}
f\left(u_{n}, u\right) & =\left(A u_{n}, u-u_{n}\right) \\
& =\left(A u_{n}-A u, u-u_{n}\right)+\left\langle A u, u-u_{n}\right\rangle .
\end{aligned}
$$

Thus,

$$
-f\left(u_{n}, u\right)=\int_{\Omega}\left(J\left(\nabla u_{n}\right)-J(\nabla u)\right)\left(\nabla u_{n}-\nabla u\right) d x+\left(A u, u_{n}-u\right) .
$$

Since $A u \in X^{*}$, then $\left(A u, u_{n}-u\right) \rightarrow 0$ as $n \rightarrow+\infty$. On the other hand from assumption $\left[\mathbf{A}_{\mathbf{1}}\right]$ and Hölder inequality, one deduces that there exist positive constants $\gamma$ and $\delta$ such that if $p \geq 2$, then

$$
\int_{\Omega}\left(J\left(\nabla u_{n}\right)-J(\nabla u)\right)\left(\nabla u-\nabla u_{n}\right) d x \geq \gamma\left\|u_{n}-u\right\|_{X}^{p}
$$

and if $1<p<2$, then

$$
\int_{\Omega}\left(J\left(\nabla u_{n}\right)-J(\nabla u)\right)\left(\nabla u-\nabla u_{n}\right) d x \geq \delta\left(\int_{\Omega}\left(\left|\nabla u_{n}\right|+|\nabla u|\right)^{p} d x\right)^{\frac{p-2}{p}}\left\|u_{n}-u\right\|_{X} .
$$

Therefore, by considering the limit sup of $-f$ in expression (4) and taking into account of $\liminf _{n} f\left(u_{n}, u\right) \geq 0$, one deduces that $u_{n} \rightarrow u$ in $X$.

Theorem 3.2. Suppose that assumptions $\left[\mathbf{A}_{\mathbf{1}}\right]-\left[\mathbf{A}_{\mathbf{4}}\right]$ are satisfied. Then for each $\lambda \in \mathbb{R}$, problem $(E V P)$ has at least one solution.

Proof. We shall apply Theorem 2.2. It is easy to see that for each $u \in K$, one has $h(u, u)=0$ and the functions $f(u, \cdot), h(u, \cdot)$ are convex. From Lemma 3.1, one has that $f$ satisfies the $(S)_{+}$condition.

Claim 1. $f(\cdot, v)$ is upper semicontinuous. 
In fact we will show that for each $v \in K$ fixed, $f(\cdot, v)$ is continuous with respect to the norm topology on $X$ and therefore upper semicontinuous on $X$. Let $\left\{u_{n}\right\}_{n \in \mathbb{N}}$ be a sequence in $K$ norm converging to $u \in K$. One has

$$
\begin{aligned}
f\left(u_{n}, v\right)-f(u, v) & =\int_{\Omega}\left[\left\langle J\left(\nabla u_{n}\right), \nabla v-\nabla u_{n}\right\rangle-\langle J(\nabla u), \nabla v-\nabla u\rangle\right] d x \\
& =\int_{\Omega}\left\langle J\left(\nabla u_{n}\right)-J(\nabla u), \nabla(v-u)\right\rangle d x+\int_{\Omega}\left\langle J\left(\nabla u_{n}\right)-J(\nabla u), \nabla\left(u_{n}-u\right)\right\rangle d x \\
& +\int_{\Omega}\left\langle J(\nabla u), \nabla u-\nabla u_{n}\right\rangle d x .
\end{aligned}
$$

Hence,

$$
\begin{aligned}
\left|f\left(u_{n}, v\right)-f(u, v)\right| \leq & \int_{\Omega}\left\|J\left(\nabla u_{n}\right)-J(\nabla u)\right\|\|\nabla(v-u)\| d x \\
& +\int_{\Omega}\left\|J\left(\nabla u_{n}\right)-J(\nabla u)\right\|\left\|\nabla\left(u_{n}-u\right)\right\| d x+\int_{\Omega}\|J(\nabla u)\|\left\|\nabla u-\nabla u_{n}\right\| d x .
\end{aligned}
$$

¿From the Hölder inequality, with $1 / p+1 / q=1$,

$$
\int_{\Omega}\left\|J\left(\nabla u_{n}\right)-J(\nabla u)\right\|\|\nabla v-\nabla u\| d x \leq\left(\int_{\Omega}\left\|J\left(\nabla u_{n}\right)-J(\nabla u)\right\|^{q} d x\right)^{\frac{1}{q}}\left(\int_{\Omega}\|\nabla v-\nabla u\|^{p} d x\right)^{\frac{1}{p}} .
$$

Taking into account of $\left[\mathbf{A}_{\mathbf{2}}\right]$, one deduces that

$$
\begin{gathered}
\int_{\Omega}\left\|J\left(\nabla u_{n}\right)-J(\nabla u)\right\|\|\nabla v-\nabla u\| d x \\
\leq \delta\left(\int_{\Omega}\left(\left\|\nabla u_{n}\right\|+\|\nabla u\|\right)^{q(p-2)}\left\|\nabla\left(u_{n}-u\right)\right\|^{q} d x\right)^{\frac{1}{q}}\|u-v\|_{X} .
\end{gathered}
$$

For $p \geq 2$, there exists a constant $\alpha_{1}>0$ such that

$$
\left(\left\|\nabla u_{n}\right\|+\|\nabla u\|\right)^{q(p-2)} \leq \alpha_{1}\left(\left\|\nabla u_{n}\right\|^{q(p-2)}+\|\nabla u\|^{q(p-2)}\right) .
$$

Therefore,

$$
\begin{array}{cl}
\int_{\Omega}\left(\left\|\nabla u_{n}\right\|+\|\nabla u\|\right)^{q(p-2)}\left\|\nabla\left(u_{n}-u\right)\right\|^{q} d x & \leq \alpha_{1}\left(\int_{\Omega}\left\|\nabla u_{n}\right\|^{q(p-2)}\left\|\nabla\left(u_{n}-u\right)\right\|^{q} d x+\right. \\
& \left.\int_{\Omega}\|\nabla u\|^{q(p-2)}\left\|\nabla\left(u_{n}-u\right)\right\|^{q} d x\right) .
\end{array}
$$

One has

$$
\begin{aligned}
\int_{\Omega}\left\|\nabla u_{n}\right\|^{q(p-2)}\left\|\nabla\left(u_{n}-u\right)\right\|^{q} d x & =\int_{\Omega}\left\|\nabla u_{n}\right\|^{\frac{p(p-2)}{p-1}}\left\|\nabla u_{n}-\nabla u\right\|^{\frac{p}{p-1}} d x \\
& \leq\left(\int_{\Omega}\left[\left\|\nabla u_{n}\right\|^{\frac{p(p-2)}{p-1}}\right]^{\alpha} d x\right)^{\frac{1}{\alpha}}\left(\int_{\Omega}\left[\left\|\nabla u_{n}-\nabla u\right\|^{\frac{p}{p-1}}\right]^{p-1} d x\right)^{\frac{1}{p-1}} \\
& \left(\frac{1}{\alpha}+\frac{1}{p-1}=1, \text { hence } \alpha=\frac{p-1}{p-2}\right) \\
& \leq\left(\int_{\Omega}\left\|\nabla u_{n}\right\|^{p} d x\right)^{\frac{1}{\alpha}}\left(\int_{\Omega}\left\|\nabla u_{n}-\nabla u\right\|^{p} d x\right)^{\frac{1}{p-1}},
\end{aligned}
$$

and

$$
\int_{\Omega}\|\nabla u\|^{q(p-2)}\left\|\nabla\left(u_{n}-u\right)\right\|^{q} d x \leq\left(\int_{\Omega}\|\nabla u\|^{p} d x\right)^{\frac{1}{\alpha}}\left(\int_{\Omega}\left\|\nabla u_{n}-\nabla u\right\|^{p} d x\right)^{\frac{1}{p-1}} .
$$

Consequently, since the sequence $\left\{u_{n}\right\}$ is bounded, there exists $\alpha_{2}>0$ such that

$$
\int_{\Omega}\left(\left\|\nabla u_{n}\right\|+\|\nabla u\|\right)^{q(p-2)}\left\|\nabla\left(u_{n}-u\right)\right\|^{q} d x \leq \alpha_{2}\left(\int_{\Omega}\left\|\nabla u_{n}-\nabla u\right\|^{p} d x\right)^{\frac{1}{p-1}}
$$


It follows, for $p \geq 2$,

$$
\int_{\Omega}\left\|J\left(\nabla u_{n}\right)-J(\nabla u)\right\|\|\nabla v-\nabla u\| d x \leq c\|u-v\|_{X}\left(\int_{\Omega}\left\|\nabla u_{n}-\nabla u\right\|^{p} d x\right)^{\frac{1}{p}},
$$

where $c>0$ is a constant. Hence

$$
\int_{\Omega}\left\|J\left(\nabla u_{n}\right)-J(\nabla u)\right\|\|\nabla v-\nabla u\| d x \leq c\|u-v\|_{X}\left\|u_{n}-u\right\|_{X} .
$$

For $1<p<2$, since $\left\|\nabla u_{n}-\nabla u\right\|^{p-2} \geq\left(\left\|\nabla u_{n}\right\|+\|\nabla u\|\right)^{p-2}$, one has

$$
\left(\left(\left\|\nabla u_{n}\right\|+\|\nabla u\|\right)^{(p-2)}\left\|\nabla\left(u_{n}-u\right)\right\|\right)^{q} \leq\left(\left\|\nabla u_{n}-\nabla u\right\|\right)^{q(p-1)} .
$$

It follows, for $1<p<2$,

$$
\begin{aligned}
\int_{\Omega}\left\|J\left(\nabla u_{n}\right)-J(\nabla u)\right\|\|\nabla v-\nabla u\| d x & \leq \delta\|u-v\|_{X}\left(\int_{\Omega}\left(\left\|\nabla u_{n}-\nabla u\right\|\right)^{q(p-1)} d x\right)^{\frac{1}{q}} \\
& \leq \delta\|u-v\|_{X}\left(\int_{\Omega}\left(\left\|\nabla u_{n}-\nabla u\right\|\right)^{p} d x\right)^{\frac{p-1}{p}} \\
& \leq \delta\|u-v\|_{X}\left\|u_{n}-u\right\|_{X}^{p-1}
\end{aligned}
$$

On the other hand,

$$
\begin{aligned}
& \int_{\Omega}\left\|J\left(\nabla u_{n}\right)-J(\nabla u)\right\|\left\|\nabla u-\nabla u_{n}\right\| d x \\
\leq & \left(\int_{\Omega}\left\|J\left(\nabla u_{n}\right)-J(\nabla u)\right\|^{q} d x\right)^{\frac{1}{q}}\left(\int_{\Omega}\left\|\nabla u-\nabla u_{n}\right\|^{p} d x\right)^{\frac{1}{p}} \\
\leq & \left\|u-u_{n}\right\|_{X}\left(\int_{\Omega}\left\|J\left(\nabla u_{n}\right)-J(\nabla u)\right\|^{q} d x\right)^{\frac{1}{q}} .
\end{aligned}
$$

One deduces in the same manner as in what precedes:

$$
\begin{gathered}
\int_{\Omega}\left\|J\left(\nabla u_{n}\right)-J(\nabla u)\right\|\left\|\nabla u-\nabla u_{n}\right\| d x \leq \delta\left\|u_{n}-u\right\|_{X}^{p}, \quad 1<p<2 ; \\
\int_{\Omega}\left\|J\left(\nabla u_{n}\right)-J(\nabla u)\right\|\left\|\nabla u-\nabla u_{n}\right\| d x \leq c\left\|u_{n}-u\right\|_{X}^{2}, \quad p \geq 2,
\end{gathered}
$$

where $c$ is a positive constant. On the other hand, from Hölder inequality, one has

$$
\int_{\Omega}\|J(\nabla u)\|\left\|\nabla u_{n}-\nabla u\right\| d x \leq\left(\int_{\Omega}\|J(\nabla u)\|^{q} d x\right)^{\frac{1}{q}}\left(\int_{\Omega}\left\|\nabla u-\nabla u_{n}\right\|^{p} d x\right)^{\frac{1}{p}} .
$$

Since $J(0)=0$ and $\|J(\nabla u)\| \leq \delta\|\nabla u\|^{p-1}$ by assumption $\left[\mathbf{A}_{\mathbf{2}}\right]$, we have

$$
\begin{aligned}
\int_{\Omega}\|J(\nabla u)\|\left\|\nabla u_{n}-\nabla u\right\| d x & \leq \delta\|u\|_{X}^{p-1}\left(\int_{\Omega}\left\|\nabla u-\nabla u_{n}\right\|^{p} d x\right)^{\frac{1}{p}} \\
& \leq \delta\|u\|_{X}^{p-1}\left\|u-u_{n}\right\|_{X} .
\end{aligned}
$$

It follows, that $f(\cdot, v)$ is continuous on $X=W^{1, p}(\Omega)$. Hence it is upper semicontinuous on $X$.

Claim 2. $\varphi(\cdot, v)$ is upper semicontinuous in $\sigma\left(X, X^{*}\right)$.

Let $\left\{u_{n}\right\}$ be a sequence in $X$ such that $u_{n} \rightarrow u$ in $\sigma\left(X, X^{*}\right)$. One has

$$
\begin{aligned}
\varphi\left(u_{n}, v\right)-\varphi(u, v)= & \lambda \int_{\Omega} \alpha(x)\left|u_{n}\right|^{p-2} u_{n}\left(v-u_{n}\right) d x-\lambda \int_{\Omega} \alpha(x)|u|^{p-2} u(v-u) d x \\
= & \lambda \int_{\Omega}\left[\alpha(x)\left|u_{n}\right|^{p-2} u_{n}-\alpha(x)|u|^{p-2} u\right]\left(v-u_{n}\right) d x \\
& +\lambda \int_{\Omega} \alpha(x)|u|^{p-2} u\left(u-u_{n}\right) d x .
\end{aligned}
$$


Hence,

$$
\begin{aligned}
\left|\varphi\left(u_{n}, v\right)-\varphi(u, v)\right| \leq & \left.|\lambda| \int_{\Omega}|\alpha(x)| u_{n}\right|^{p-2} u_{n}-\alpha(x)|u|^{p-2} u|| v-u_{n} \mid d x \\
& +\left.|\lambda| \int_{\Omega}|\alpha(x)| u\right|^{p-2} u|| u-u_{n} \mid d x .
\end{aligned}
$$

One has by Hölder inequality, with $\frac{1}{q^{*}}+\frac{1}{p^{*}}=1$,

$\left.\int_{\Omega}|\alpha(x)| u_{n}\right|^{p-2} u_{n}-\alpha(x)|u|^{p-2} u|| v-u_{n}\left|d x \leq\left\|\alpha(x)\left|u_{n}\right|^{p-2} u_{n}-\alpha(x)|u|^{p-2} u\right\|_{L^{q^{*}}}\left\|v-u_{n}\right\|_{L^{p^{*}}}\right.$

Since $X$ is compactly embedded in $L^{p^{*}}(\Omega)$ we may again assume $u_{n} \rightarrow u$ in $L^{p^{*}}(\Omega)$. Therefore, by Krasnoselskii [18, p.30],

$$
\alpha(x)\left|u_{n}\right|^{p-2} u_{n} \rightarrow \alpha(x)|u|^{p-2} u \text { in } L^{q^{*}}(\Omega) .
$$

On the other hand,

$$
\left.\int_{\Omega}|\alpha(x)| u\right|^{p-2} u\left\|u-u_{n}\left|d x \leq\left\|\alpha(x)|u|^{p-2} u\right\|_{L^{q^{*}}}\left\|u-u_{n}\right\|_{L^{p^{*}}}\right.\right.
$$

Since $\left\|u-u_{n}\right\|_{L^{p^{*}}} \rightarrow 0$, one deduces that

$$
\left.\int_{\Omega}|\alpha(x)| u\right|^{p-2} u|| u-u_{n} \mid d x \rightarrow 0 .
$$

Consequently, one deduces that $\varphi(\cdot, v)$ is continuous in the weak topology of $X$ for each $\lambda \in \mathbb{R}$. Therefore, $\varphi(\cdot, v)$ is $\sigma\left(X, X^{*}\right)$-upper semicontinuous for each $\lambda \in \mathbb{R}$.

Claim 3. $\psi(\cdot, v)$ is upper semicontinuous in $\sigma\left(X, X^{*}\right)$.

Let $\left\{u_{n}\right\}$ be a sequence in $X$ such that $u_{n} \rightarrow u$ in $\sigma\left(X, X^{*}\right)$. Then

$$
\begin{aligned}
\psi\left(u_{n}, v\right)-\psi(u, v) & =\int_{\Omega} \theta\left(x, u_{n}\right)\left(v-u_{n}\right) d x-\int_{\Omega} \theta(x, u)(v-u) d x \\
& =\int_{\Omega}\left(\theta\left(x, u_{n}\right)-\theta(x, u)\right)\left(v-u_{n}\right) d x+\int_{\Omega} \theta(x, u)\left(u-u_{n}\right) d x .
\end{aligned}
$$

One has from the Hölder inequality

$$
\int_{\Omega}\left|\theta\left(x, u_{n}\right)-\theta(x, u)\right|\left|v-u_{n}\right| d x \leq\left\|\theta\left(x, u_{n}\right)-\theta(x, u)\right\|_{L^{q^{*}}}\left\|v-u_{n}\right\|_{L^{p^{*}}}
$$

Since $X$ is compactly embedded in $L^{p^{*}}(\Omega)$, we may again assume $u_{n} \rightarrow u$ in $L^{p^{*}}(\Omega)$ and, by an argument in Krasnoselskii [18, p.30], $\left\|\theta\left(x, u_{n}\right)-\theta(x, u)\right\|_{L^{q^{*}}} \rightarrow 0$. Hence

$$
\int_{\Omega}\left|\theta\left(x, u_{n}\right)-\theta(x, u)\right|\left|v-u_{n}\right| d x \longrightarrow 0
$$

On the other hand

$$
\int_{\Omega}\left|\theta(x, u)\left\|u-u_{n} \mid d x \leq\right\| \theta(x, u)\left\|_{L^{q^{*}}}\right\| u-u_{n} \|_{L^{p^{*}}}\right.
$$


Since $\left\|u-u_{n}\right\|_{L^{p^{*}}} \rightarrow 0$, we have $\int_{\Omega}|\theta(x, u)|\left|u-u_{n}\right| d x \rightarrow 0$. Consequently, $\psi(\cdot, v)$ is continuous in the weak topology on $X$. As a result, it is $\sigma\left(X, X^{*}\right)$-upper semicontinuous.

Claim 4. The Coercivity condition is valid.

Let $v_{0}$ in $K$ be fixed. One has

$$
f\left(u, v_{0}\right)=\|u\|_{X}^{p+1} \int_{\Omega}\left\langle J\left(\nabla\left(\|u\|_{X}^{-1} u\right)\right), \nabla\left(\|u\|_{X}^{-1} v_{0}-\|u\|_{X}^{-1} u\right)\right\rangle d x,
$$

therefore

$$
\frac{f\left(u, v_{0}\right)}{\|u\|_{X}^{p}}=\int_{\Omega}\left\langle J\left(\nabla\left(\|u\|_{X}^{-1} u\right)\right), \nabla v_{0}\right\rangle d x-\|u\|_{X} \int_{\Omega}\left\langle J\left(\nabla\left(\|u\|_{X}^{-1} u\right)\right), \nabla\left(\|u\|_{X}^{-1} u\right)\right\rangle d x
$$

It is easy to verify that

$$
\left|\int_{\Omega}\left\langle J\left(\nabla\left(\|u\|_{X}^{-1} u\right)\right), \nabla v_{0}\right\rangle d x\right| \leq \delta\left\|v_{0}\right\|_{X}
$$

and

$$
0 \leq \int_{\Omega}\left\langle J\left(\nabla\left(\|u\|_{X}^{-1} u\right)\right), \nabla\left(\|u\|_{X}^{-1} u\right)\right\rangle d x \leq \delta .
$$

It follows from relations $(6),(7)$ and (8) that

$$
\lim _{\|u\|_{X} \rightarrow+\infty} \frac{f\left(u, v_{0}\right)}{\|u\|_{X}^{p}}=-\infty .
$$

On the other hand, one has

$$
\varphi\left(u, v_{0}\right)=\lambda\|u\|_{X}^{p} \int_{\Omega} \alpha(x)\left|\frac{u}{\|u\|_{X}}\right|^{p-2} \frac{u}{\|u\|_{X}}\left(\|u\|_{X}^{-1} v_{0}-\frac{u}{\|u\|_{X}}\right) d x
$$

Hence

$$
\begin{aligned}
\frac{\left|\varphi\left(u, v_{0}\right)\right|}{\|u\|_{X}^{p}} & \leq|\lambda| \frac{1}{\|u\|_{X}}\left\|v_{0}-u\right\|_{L^{p^{*}}(\Omega)}\left\|\left.\left.\alpha(x)\right|_{\frac{u}{\|u\|_{X}}}\right|^{p-2} \frac{u}{\|u\|_{X}}\right\|_{\left(L^{p^{*}}(\Omega)\right)^{\prime}} \\
& \leq|\lambda| \frac{1}{\|u\|_{X}}\left\|v_{0}-u\right\|_{L^{p^{*}}(\Omega)}\|\alpha(x)\|_{L^{\frac{N}{p}}(\Omega)}\left\|\frac{u}{\|u\|_{X}}\right\|_{L^{p^{*}}(\Omega)} \\
& \leq c|\lambda|\|\alpha(x)\|_{L^{\frac{N}{p}}(\Omega)} \frac{\left\|v_{0}-u\right\|}{\|u\|_{X}}
\end{aligned}
$$

where $c$ is a positive constant. It follows that $\frac{\left|\varphi\left(u, v_{0}\right)\right|}{\|u\|_{X}^{p}}$ is bounded when $\|u\|_{X} \rightarrow+\infty$. One can see in the same manner that $\frac{\left|\psi\left(u, v_{0}\right)\right|}{\|u\|_{X}^{p}}$ is bounded when $\|u\|_{X} \rightarrow+\infty$. Consequently, $\frac{f\left(u, v_{0}\right)+h\left(u, v_{0}\right)}{\|u\|_{X}^{p}} \rightarrow-\infty$ when $\|u\|_{X} \rightarrow+\infty$. Therefore, there exists $R>0$ such that

$$
f\left(u, v_{0}\right)+h\left(u, v_{0}\right)<0 \text { for } u \in K \text { and }\|u\|_{X}>R .
$$

Hence the coercivity assumption $(v)$ of Theorem 2.2 is satisfied. This completes the proof.

\section{Remarks and comments.}


1. Note that the same results can be obtained in the case where $g$ is of the form :

$$
g(x, u, \lambda)=\lambda \rho(x)|u|^{\gamma-2} u+\theta(x, u),
$$

where $\rho(x) \in L^{\frac{p^{*}}{p^{*}-\gamma}}(\Omega), 1<\gamma<p$ and $\theta$ is of the same form considered in the paper.

2. The operator $A$ in the eigenvalue problem $(E V P)$ is not bounded. As a result, results in [12] and [10] are not applicable in solving $(E V P)$.

3. The technique developed in this paper and its application to the eigenvalue problem $(E V P)$ is more simpler in comparison with other techniques used in e.g. [21, 19, 22, 20, $23,19,25]$. This leads us to obtain existence of solutions independently of $\lambda \in \mathbb{R}$ which is not the case in some of the cited works where the existence of solutions depends on the value of $\lambda$ in a bounded interval. Our tools can be applied and simplify the study of many problems from mechanics, engineering and others which formulations can be written as an equilibrium problem.

\section{References}

[1] Ansari, Q.H., Wong, N.-C., and Yao, J.-C (1999). The existence of Nonlinear Inequalities, Applied Mathematics Letters, 12 89-92.

[2] Aubin, J. P. and Ekeland, I. (1984). Applied Nonlinear Analysis. John Wiley and Sons.

[3] Baek, J. S. (1992). Properties of Solutions to a Class of Quasilinear Elliptic Problems. Ph.D. dissertation, Seoul National University.

[4] Bianchi, M. and Schaible, S. (1996). Generalized Monotone Bifunctions and Equilibrium Problems. J. Optim. Theory Appl. 90, 31-43.

[5] Blum, E. and Oettli, W. (1994). From Optimization and Variational Inequalities to Equilibrium Problems. The Mathematics Student 63, 123-145.

[6] Browder, F.E. (1968). Nonlinear eigenvalue problems and Galerkin approximations, Bull. Amer. Math. Soc. 74, 651-656.

[7] Chadli, O. and Yao, J.C. Regularized Equilibrium Problems and Application to Noncoercive Hemivariational Inequalities. Preprint.

[8] Chadli, O., Chbani, Z. and Riahi, H. (2000). Equilibrium Problems with Generalized Monotone Bifunctions and Applications to Variational Inequalities. J. Optim. Theory Appl. 105, 299-323.

[9] Chadli, O., Chbani, Z. and Riahi, H. (1999). Recession Methods for Equilibrium Problems and Applications to Variational and Hemivariational Inequalities. Discrete and Continuous Dynamical Systems 5, 185-195.

[10] Cubiotti, P. (1997). General nonlinear Variational inequalities with $(S)_{+}^{1}$ operators, Appl. Math. Lett. 10, 11-15. 
[11] Cubiotti, P. and Yao J.-C. (1995). Multivalued $(S)_{+}^{1}$ operators and generalized variational inequalities, Computers math. Applic. 29, 49-56.

[12] Ding, X.P. and Tarafdar, E. (1995). Existence and uniqueness of solutions for a general nonlinear variational inequality, Appl. Math. Lett. 8, 31-36.

[13] Fan, K. (1961). A generalization of Tychonoff's fixed point theorem. Mathematishe Annalen 142, 305-310.

[14] Hadjisavvas, N. and Schaible, S. (1996). Quasimonotone Variational Inequalities in Banach Spaces. J. Optim. Theory Appl. 90, 55-111.

[15] Isac, G. and Gowda, M.S., (1993). Operators of $(S)_{+}^{1}$, Altman's condition and the complementarity problem, J. Fac. Sci. Univ. Tokyo, Sect. IA, Math.40, 1-16.

[16] Konnov, I.V. and Yao, J.C. (1997). On the Generalized Vector Variational Inequality Problem. J. Math. Anal. Appli. 206, 42-58.

[17] Köthe, G. (1969) Topological Vector Space I, Spinger-Berlag, Berlin.

[18] Krasnoselskii, M.A. (1964). Topological Methods In The Theory Of Nonlinear Integral Equations, Pergamon, Elmosford, NY.

[19] Le, V. K. (1999). Some Degree Calculations and Applications to Global Bifurcation Results of Variational Inequalities. Nonlinear Anal. 37, 473-500.

[20] Schuricht, F. (1996). Bifurcation from Minimax Solutions by Variational Inequalities in Convex Sets. Nonlinear Anal. 26, 91-112.

[21] Szulkin, A. (1986). Existence and Nonuniqueness of Solutions of a Noncoercive Elliptic Variational Inequality. Proc. Sympos. Pure Math. 45, 413-418.

[22] Yang, J. F. (1995). Positive Solutions of Quasilinear Elliptic Obstacle Problems with Critical Exponents. Nonlinear Anal. 25, 1283-1306.

[23] Yang, J. F. (1997). Regularity of Weak Solutions to Quasilinear Elliptic Obstacle Problems. Acta Math. Sci. 17, 159-166.

[24] Zhow, J. X. and Chen, G. (1988). Diagonal convexity conditions for problems in convex analysis and quasi-variational inequalities, J. Math. Appli. Anal. 132, 213-225.

[25] Zhou, Y. and Huang, Y. (2000). Existence of Solutions for a Class of Elliptic Variational Inequalities. J. Math. Anal. Appl. 250, 187-195.

[26] Zhou, Y. and Huang, Y. (1996). Several Existence Theorems for the Nonlinear Complementarity Problems. J. Math. Anal. Appl. 202, 776-784. 